This item was submitted to Loughborough's Research Repository by the author.

Items in Figshare are protected by copyright, with all rights reserved, unless otherwise indicated.

\title{
Deterministic modeling of the diffusive memristor
}

PLEASE CITE THE PUBLISHED VERSION

https://doi.org/10.1063/5.0056239

PUBLISHER

AIP Publishing

VERSION

AM (Accepted Manuscript)

PUBLISHER STATEMENT

This article may be downloaded for personal use only. Any other use requires prior permission of the author and AIP Publishing. This article appeared in Akther, A. .. et al., (2021). Deterministic modeling of the diffusive memristor. Chaos 31, 073111 and may be found at https://doi.org/10.1063/5.0056239

\section{LICENCE}

All Rights Reserved

\section{REPOSITORY RECORD}

Akther, Amir, Yury Ushakov, Alexander Balanov, and Sergey Saveliev. 2021. "Deterministic Modeling of the Diffusive Memristor". Loughborough University. https://hdl.handle.net/2134/14818512.v1. 


\title{
Deterministic modeling of the diffusive memristor
}

\author{
A. Akther,Corresponding author: A.Akther@lboro.ac.uk, ${ }^{1}$ Y. Ushakov, ${ }^{1}$ A.G. Balanov, ${ }^{1}$ and S.E. Savel'ev ${ }^{1}$ \\ Department of Physics, Loughborough University LE11 3TU, UK
}

(Dated: 15 June 2021)

Recently developed diffusive memristors have gathered a large amount of research attention due to their unique property to exhibit a variety of spiking regimes reminiscent to that found in biological cells, which creates a great potential for their application in neuromorphic systems of artificial intelligence and unconventional computing. These devices are known to produce a huge range of interesting phenomena through the interplay of regular, chaotic and stochastic behavior. However, the character of these interplays as well as the instabilities responsible for different dynamical regimes are still poorly studied, because of the difficulties in analyzing the complex stochastic dynamics of the memristive devices. In this paper we introduce a new deterministic model justified from the Fokker-Planck description to capture the noise-driven dynamics that noise has been known to produce in the diffusive memristor. This allows us to apply bifurcation theory to reveal the instabilities and the description of the transition between the dynamical regimes.

\begin{abstract}
Recently fabricated diffusive memristors ${ }^{1}$ show a wide range of complex dynamics determined by the interplay of nanomechanical, heat, and electric degrees of freedom. If the diffusive memristor is connected to a simple circuit with a resistor in series and a capacitor in parallel, such a neuromorphic circuit demonstrates different types of oscillations, some of which are driven by dynamical features of the system and others are induced by noise. More interestingly, the system starts to demonstrate a new class of emerging phenomena which can only appear due to the interplay of dynamical and noise-driven properties. To understand and control the system we need to develop models which uncover such emerging mechanisms, this can be done by proper averaging over the stochastic dynamics and separating fast and slow degrees of freedom within the system which is the goal of this paper.
\end{abstract}

\section{INTRODUCTION}

For the past decade artificial intelligence and machine learning has witnessed a huge increase in their applications and their utilization in both academia and industry, with modern machine learning methods being applied in a huge range of fields, such as financial economics ${ }^{2}$, particle physics ${ }^{3,4}$ and now even autonomous vehicles ${ }^{5,6}$. However with increasing applications and a constant increase to the size of the data needed to run such models we have begun to see the limits of modern computational architecture, such as an ever growing need for greater power consumption along with relying on unreasonably long time scales to complete such tasks. We have grown accustom to constant improvements to computing power and the consistent miniaturization of computational components $^{7}$, to sustain this pattern it is now clear that we need a new form of computing. Fortunately, a great area of promise is within that of neuromorphic computing, which attempts to replicate the information processing mechanisms found in biological circuits comprised of neurons that enable rapid pattern recognition at an extraordinarily low power consumption. The first milestone in this direction is to produce an artificial memristor-based neuron which has the same level of plasticity that biological neurons have, or even to outperform them. These artificial neurons then allow for the development of hardware-based spiking neural network computing, which is close to impossible for current software implementation due to resource constraints.

In $1971 \mathrm{Chua}^{8}$ published the seminal paper in which he postulated that there should be an additional, fourth fundamental circuit element. In observing that there was a form of symmetry between the interacting relationships of the four circuit variables, specifically: the current $i$, the charge $q$, the voltage $v$ and the flux linkage $\varphi$, there should be an additional circuit element linking flux linkage and charge. This new element was named a "memristor". In a future paper Chua and Kang 9 expanded on this original idea and introduced a new class of dynamical systems which was called "memrisitive systems". Whilst the idea has been around since 1971, it was not until 2008 when HP labs ${ }^{10}$ had experimentally verified that the memristor did have a physical counterpart to its theoretical existence. The memristor is a two terminal device which has the unique property that its resistance is dependent on some internal variable which can change when a current flowing through the memristor that is reminiscent to the dynamics of a biological neuron and synapse.

Since the experimental discovery of the memristor there has been an emergence of different types of devices which fall under this category. For example, the so-called nonvolatile memristor ${ }^{11}$ has the property of being able to maintain either the high- or low-resistive state after the external voltage has been removed $^{12}$. The other recently developed volatile memristors switch back to the same resistive state (usually with high resistance) after a short relaxation time of the order of milliseconds after the external voltage has been removed (see, e.g., Ref. ${ }^{1}$ ). The combination of volatile and non-volatile memristors allows for the realization of both artificial synapses and artificial neurons for more accurate mimicking of the behaviors observed in neural networks and therefore may be more suitable for the desired goal of neuromorphic computing (see, e.g., Ref. $\left.{ }^{13}\right)$.

There have been many devices produced which would classify as a volatile memristor, of which they rely on different physical mechanisms for resistive switching ${ }^{14-16}$. A new form of volatile memristor has been fabricated and is called the dif- 
fusive memristor. This device produces resistive switching through the diffusion of $\mathrm{Ag}$-metallic nanoclusters within $\mathrm{SiO}_{2}$ from one terminal to another, this forms a bridge between the two terminals ${ }^{1}$. The combination of mechanical, thermal and electrical degrees of freedom allows this device to have a wide range of stochastic, dynamical and sometimes chaotic behavior (see, e.g., $\operatorname{Ref}^{17,18}$ ). Not only is this memristor interesting from a mathematical viewpoint, the device can emulate both long-term and short-term plasticity ${ }^{1}$, meaning that it resembles biological cell mechanics closer than other memristive devices.

"Artificial neurons" based on different memristors have been produced ${ }^{19-22}$, of which they are seemingly capable of showing regular spiking which makes them an ideal candidate for oscillator-based computing ${ }^{23,24}$, a new computational concept relying on accurate phase and frequency locking of synchronized oscillators. This however is problematic for the diffusive memristor as this device shows highly random spiking, but this may be advantageous when mimicking biological neurons where noise plays an essential role ${ }^{25}$.

Even though the end goal for memristor research is to analyze large ensembles of interacting devices it should be noted that each individual part of the memristive network should be investigated. Within this paper we analyze a single artificial neuron based on the same diffusive memristor within ${ }^{17,26}$, but instead of performing stochastic simulations, we formulate the corresponding Fokker-Planck equation for a diffusing $A g$ cluster and solve it by expanding the potential for the cluster up to the second order with respect to the particles deviation from its average trajectory. We then investigate the new system of ordinary differential equations to see what deterministic mechanisms of spiking the system exhibits. We find that the deterministic model is capable of replicating the two main forms of spiking observed in the stochastic version, providing us with a better understanding of how to control the seemingly random spiking of stochastic diffusive artificial neurons.

\section{MODELS AND METHODS}

\section{A. Memristor model}

The memristor which has been investigated in this section will be a two-terminal device where $A g$ clusters (which we can also call particles) may travel from one terminal to another and the distribution of these clusters will intern define the resistance of the memristor. A simple model which considers cluster diffusion coupled to the heat and electron dynamics $1,13,26,27$ can provide an accurate description of electric circuits with diffusive memristors. In a simplified version of that model for an artificial neuron circuit (see Fig. 1(a)), where only one $A g$ cluster is considered, is still powerful enough to capture most experimental features and simultaneously allows to quantitatively interpret the obtained results ${ }^{17}$. The model is (a)

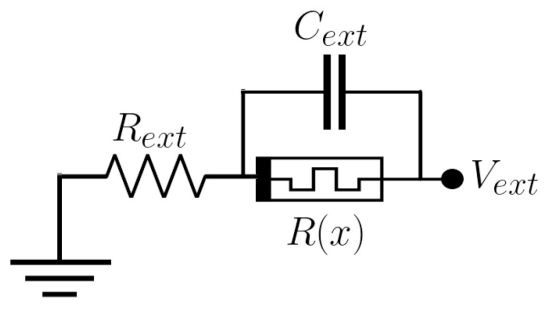

(b)

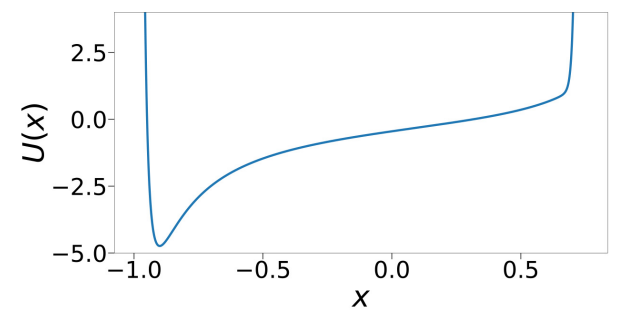

FIG. 1: a) The circuit diagram of the model given by Eq. (1). b) The memristor potential profile $U(x)$ given by Eq. (2).

given:

$$
\left\{\begin{array}{l}
\eta \frac{d x}{d t}=-U^{\prime}(x)+q V+\sqrt{\eta T} \xi(t), \\
\frac{d T}{d t}=\frac{V^{2}}{\gamma R(x)}-\kappa\left(T-T_{0}\right), \\
\tau_{c} \frac{d V}{d t}=V_{e x t}-\left(1+\frac{R_{e x t}}{R(x)}\right) V .
\end{array}\right.
$$

Here we have $x$ defining the position of the particle, $q$ is the electrical charge of the $A g$ cluster, $V$ is the voltage between the two terminals, $\mathrm{T}$ is the temperature of the device, $\xi(t)$ is a zero-averaged $\delta$-correlated Gaussian noise, and $\eta$ is the effective viscosity of the $\mathrm{Ag}$ clusters in the $\mathrm{SiO}_{2}$ matrix. The first of the three equations describes the motion of the particle in a tilted potential $U(x)$ under the influence of thermal noise and an externally applied electric field. We have used ' to denote the derivative with respect to $x$.

We model the potential as such:

$$
\eta U(x)=\frac{0.05}{(x+1)^{2}}-\frac{1}{x+1}-\frac{0.5}{x-1}+(1.2 x+0.168)^{100} .
$$

The contribution to this potential comes through (i) interfacial energy ${ }^{26}$ favoring the cluster to be attracted to other non-movable clusters, forming filaments ${ }^{28,29}$ or memristor terminals, (ii) interaction of the particle with inhomogeneities/pinning ${ }^{30}$ and insulating matrix ${ }^{31}$. The shape of $U(x)$ (Fig. 1(b)) was motivated by the experimental observation of hysteresis in current-voltage curves in both currentand voltage-driven regimes of volatile memristors ${ }^{32-34}$, which means the co-existence of $S$ - and $N$-types of negative differential resistance within the same current-voltage characteristic of a device (a similar property was found in superconductors $^{35}$ ). This is an important peculiarity of memristors, which 
makes them capable of generating different types of current pulses $^{36}$.

In order to reproduce that hysteretic behavior, the combination of the above mentioned forces should result in a potential with a negative second order derivative inside a region between the memristor terminals to prohibit an adiabatic motion of the cluster when driven by the constant voltage; the potential we consider here has such a region of a negative second derivative. We found it appropriate to use the classical model of the central field force potential ${ }^{37}$, which forms an attracting well next to the left memristor terminal (close to $x=-1$ ), whilst the particle cannot reach the right terminal due to a strong repulsive pinning (modeled by the last term of Eq. (2)) discussed in $\operatorname{Ref}^{30}$.

The second equation in the system (1) models the heat exchange $^{38}$ within the device with the Joule heat source which is controlled by the memristors resistance $R(x)$ and heat capacitance $\gamma$, the heat sink is determined by the heat-transfer coefficient $\kappa$ and the bath temperature $T_{0}$.

The third equation is the Kirchhoff's rule for the circuit under consideration Fig. 1(a), where we define external resistance with $R_{e x t}$, external voltage with $V_{e x t}$, and so-called $R C$ time, $\tau_{c}$, of the capacitor charging.

The resistance of the memristor is dependent on the current position of the particle. We assume that given the particle fluctuates between the two electrodes, and that the electrons tunnel from one terminal to the other through the particle (somewhat similar to electron shuttling, see e.g., Ref. ${ }^{39}$ ), this results in a fluctuating resistance. The tunneling resistance between one of the electrodes and the particle, and the second electrode and the particle are, respectively, $R_{1} \propto \exp [(1-x) / \lambda]$ and $R_{2} \propto \exp [(1+x) / \lambda]$, where we define $\lambda$ (see, e.g., Ref. ${ }^{40}$ ) to equal the tunneling length, which implies that all resistances are normalized by the lowest resistance of the memristor. This allows us to rewrite the total resistance as $R(x)=$ $R_{1}+R_{2}=R_{0} \cosh (x / \lambda)$, below we will normalize all resistances to $R_{0}=1$, that is $R_{0}$ is our unit for resistance. Note that the current through the memristor can be calculated using Ohms law, $I=V / R(x)$. The circuit shown in Fig. 1(a) is called an "artificial neuron" 22 within the literature because it has the capability to produce short impulses which can be viewed in a similar manner as the spiking which is observed in biological cells.

\section{B. Deterministic Model}

In a series of works performed by the research group of V. S. Anishchenko and their collaborators it was demonstrated that certain stochastic phenomena can be observed in purely deterministic chaotic systems $\mathrm{s}^{41,42}$, while the effects typical for the deterministic oscillators can also occur in systems with noise-induced dynamics ${ }^{43-45}$. Partly, such universality of the phenomena can be explained by the fact that often noisy dynamics and deterministic chaos in dynamical systems demonstrate similar statistical properties ${ }^{46,47}$. On the other hand, noise can induce a distinct time scale in the system, which makes the dynamics quite coherent. Moreover, under certain condition small random fluctuations are able to evoke the dynamics demonstrating the properties of deterministic chaos ${ }^{48}$. All these findings suggest that under certain assumptions the interplay between stochastic and deterministic phenomena allows for a deterministic description, which could simplify the analysis.

In our case we start with by considering the Fokker-Planck equation corresponding to the model (1), which is a partial differential equation in $4 \mathrm{D}(t, x, V, T)$ space. For simplicity we consider the situation when temperature and voltage changes slowly compared to particle diffusion. In this case, we can state that both temperature and voltage can be described by ordinary differential equations with an average conductance $\langle 1 / R(x)\rangle$ determined by a reduced Fokker-Planck equation for the particle diffusion in the $(t, x)$ space, namely:

$$
\left\{\begin{array}{l}
\frac{d T}{d t}=\frac{V^{2}}{\gamma}\left\langle\frac{1}{R(x)}\right\rangle-\kappa\left(T-T_{0}\right), \\
\frac{d V}{d t}=\frac{1}{\tau_{c}}\left[V_{e x t}-\left(1+R_{e x t}\left\langle\frac{1}{R(x)}\right\rangle\right) V\right], \\
\eta \frac{\partial P}{\partial t}=-\frac{\partial}{\partial x}\left[-U^{\prime}(x)+q V\right] P+T \frac{\partial^{2} P}{\partial x^{2}} .
\end{array}\right.
$$

We have used the angled brackets to represent the averaged value. If we now approximate the derivative of the potential as: $U^{\prime}(x)=U^{\prime}\left(x_{0}\right)+U^{\prime \prime}\left(x_{0}\right)\left(x-x_{0}\right)$. Then we shall seek a solution of the Fokker-Planck equation in the form: $P=A \frac{1}{\sqrt{\Delta}} e^{-\frac{\left(x-x_{0}\right)^{2}}{\Delta}}$, with two new variables $x_{0}(t)$ and $\Delta(t)$, which describes the particle's average position and the standard deviation of thermal fluctuations of the particle's motion. This then lets us have four ordinary differential equations:

$$
\left\{\begin{array}{l}
\eta \frac{d x_{0}}{d t}=-U^{\prime}\left(x_{0}\right)+q V, \\
\eta \frac{d \Delta}{d t}=4 T-2 \Delta U^{\prime \prime}\left(x_{0}\right), \\
\frac{d T}{d t}=\frac{V^{2}}{\gamma}\left\langle\frac{1}{R(x)}\right\rangle-\kappa\left(T-T_{0}\right), \\
\frac{d V}{d t}=\frac{1}{\tau_{c}}\left[V_{e x t}-\left(1+R_{e x t}\left\langle\frac{1}{R(x)}\right\rangle\right) V\right] .
\end{array}\right.
$$

Where

$$
\left\langle\frac{1}{R(x)}\right\rangle=\frac{A}{\sqrt{\Delta}} \int_{-\infty}^{\infty} \frac{1}{\cosh \left(\frac{x}{\lambda}\right)} e^{-\frac{\left(x-x_{0}\right)^{2}}{\Delta}} d x
$$

To estimate this integral we approximate $1 / \cosh (x / \lambda)$ for the particle located between memristor electrodes $-1<x<1$ (with $x= \pm 1$ corresponding to the left/right electrode and $x$ normalized by half of the distance between electrodes) by a sum,

$$
\frac{1}{\cosh \left(\frac{x}{\lambda}\right)} \approx \sum P_{i} e^{-b_{i} x^{2}}
$$

By using the least squares regression for four terms in the sum (6) and $\lambda=0.12$, we estimate the parameters $P_{i}$ and $b_{i}$ as: $P_{1}=0.1398, P_{2}=0.4306, P_{3}=0.3509, P_{4}=0.0785 ; b_{1}=$ 


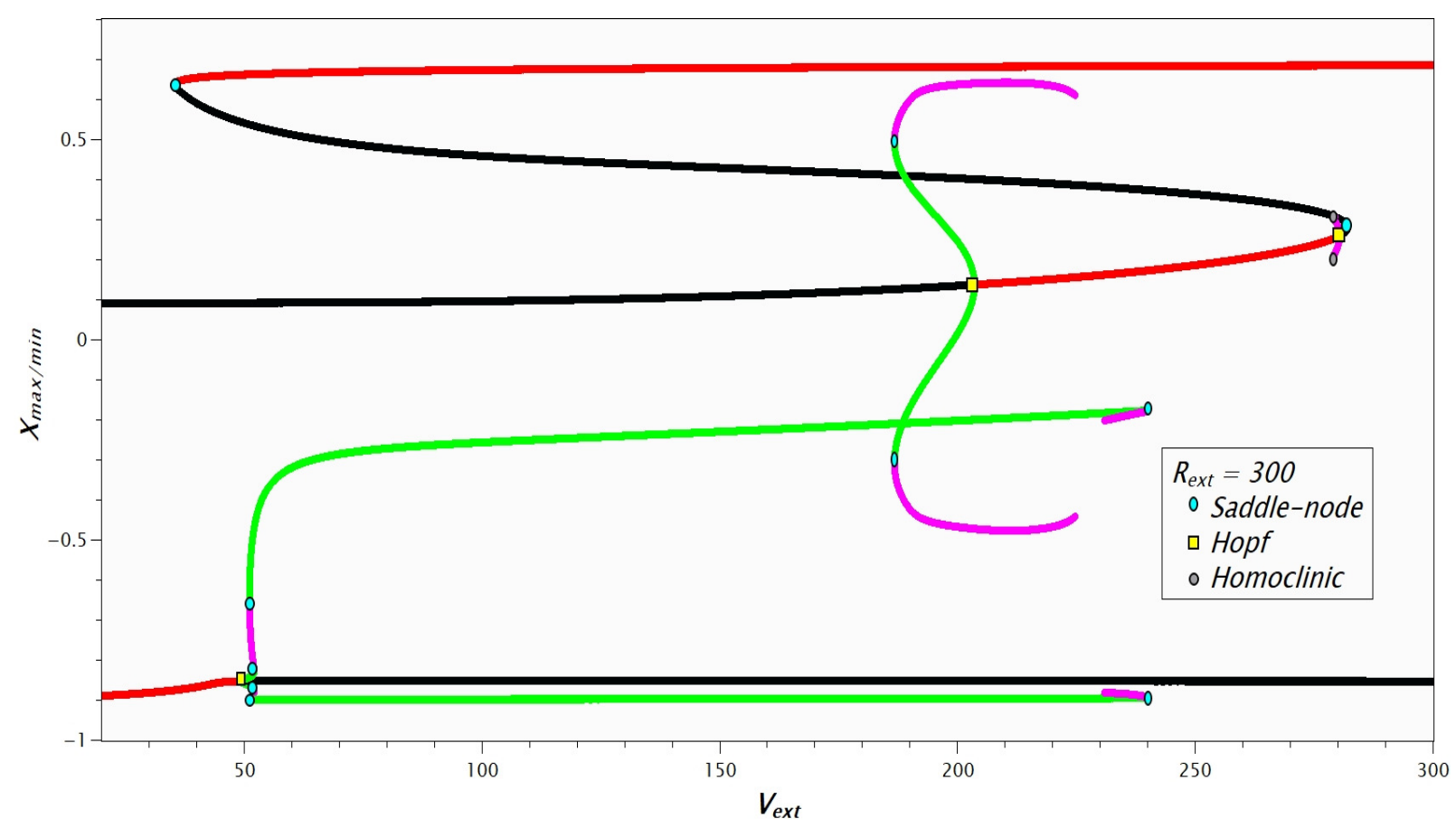

FIG. 2: One-dimensional bifurcation diagram (at $R_{\text {ext }}=300$ ). The red color indicates the stable fixed point, black is for an unstable one, green and pink are for maximum and minimum values of $x_{0}$ on stable and unstable limit cycles, respectively.

95.3853, $b_{2}=36.8889, b_{3}=14.0560, b_{4}=5.3923$. Now evaluating the integral (5) leads to

$$
\left\langle\frac{1}{R(x)}\right\rangle=\sum P_{i} e^{-\frac{b_{i} x_{0}^{2}}{1+b_{i} \Delta}} \frac{1}{\sqrt{1+b_{i} \Delta}} .
$$

Substituting (7) into (4) allows us to derive the set of equations that we will study in this paper to model the artificial neuron

$$
\left\{\begin{array}{l}
\frac{d x_{0}}{d t}=M\left(-U^{\prime}\left(x_{0}\right)+q V\right), \\
\frac{d \Delta}{d t}=M\left(4 T-2 \Delta U^{\prime \prime}\left(x_{0}\right)\right) \\
\frac{d T}{d t}=\frac{V^{2}}{\gamma} \sum P_{i} e^{-\frac{b_{i} x_{0}^{2}}{1+b_{i} \Delta}} \frac{1}{\sqrt{1+b_{i} \Delta}}-\kappa\left(T-T_{0}\right), \\
\tau_{c} \frac{d V}{d t}=V_{e x t}-\left(1+R_{e x t} \sum P_{i} e^{-\frac{b_{i} x_{0}^{2}}{1+b_{i} \Delta}} \frac{1}{\sqrt{1+b_{i} \Delta}}\right) V
\end{array}\right.
$$

with $M=1 / \eta$.

Below, numerical simulations of the system (8) are carried out by through use of the LSODA package ${ }^{49}$ with step size $t=$ 0.0001 . The numerical simulations for the oscillations are ran with the same values used for the bifurcation analysis: $M q=$ $0.50, \tau_{c}=2, R_{\text {ext }}=300, M=0.3, \gamma=16.7, \kappa=0.9$ and $T_{0}=0$. For numerical simulations relating to the figures, Fig. 3 and Fig. 4, we fix voltage to $V_{\text {ext }}=186.8$.

\section{SPIKING REGIMES}

Within the artificial neuron model Eq. (1) there are two different spiking regimes ${ }^{17}$, one associated with charge/discharge cycles and the other being produced due to a much slower heating-cooling cycle caused by thermal effects. The former spiking phenomena can be explained as such, when the external voltage supply $V_{\text {ext }}$ is originally switched on, the memristor is at a high resistive state and the capacitor begins to charge due to the current flowing through it. However at a certain voltage, $V$, is great enough to force the particle to escape the potential well and starts to approach the center point of the device and therefore the resistance, $R(x)$, enters its lowest value. This then causes the capacitor to discharge leading to voltage decreasing, resistance then begins to increase once more and the particle re-enters the well and this results in the capacitor to start recharging, resembling the Pearson-Anson effect ${ }^{50}$.

The second spiking regime can only be achieved via the interplay of stochastic and deterministic dynamics in the original model (1) and is associated with the particle being moved due to thermal effects within the device, going through heating and cooling cycles. Previous works have shown that this regime involves noise-induced phenomena ${ }^{17}$, however the dynamical mechanisms and related instabilities capable to evoke such spiking behavior still remain unclear. To investigate these open questions we utilize the model (8). 


\section{BIFURCATION ANALYSIS AND NUMERICAL RESULTS}

The analysis of the mechanisms governing the emergence of the spiking phenomena in the model (8), which describes the circuit in Fig. 1(a), is summarized in the bifurcation diagram presented in Fig. 2. Here, the y-axis is representing the maximum and minimum values of the position, $x_{0}$, and the horizontal axis is representing the external voltage, $V_{\text {ext }}$.

The bifurcation diagram in Fig. 2 reveals three branches of the fixed points (red and black points) which give rise to the stable solutions. They correspond to the positions, $x_{0}$ close to -0.9 (lower branch), 0 (middle branch) and 0.6 (upper branch). The coexistence of these branches for the same values of $V_{\text {ext }}$ can explain the hysteretic transitions in the current-voltage characteristics and the multistability measured previously in diffusive memristors (see, e.g., Ref. ${ }^{27}$ ) For low voltages $V_{\text {ext }}$ the system demonstrate the only stable solution, which is a lower branch equilibrium (red point) at $x_{0}$ close to -0.9 corresponding to the potential minimum of $U(x)$, see Fig. 1(b). The fixed point remains stable until an Andronov-Hopf bifurcation at $V_{e x t} \approx 49.1$, where it loses its stability, and then remains unstable for larger $V_{\text {ext }}$ (black points). The Andronov-Hopf bifurcation gives birth to a stable periodic orbit, whose extreme values of $x$ are shown by green points. These small-amplitude stable oscillations rapidly disappear due to a saddle-node bifurcation at $V_{\text {ext }} \approx 51.9$, which implies a collision of a stable cycle (green points) with an unstable limit cycle (pink points). Another saddle-node bifurcation for limit cycles leads to the reappearance of stable self-oscillations at $V_{e x t} \approx 51.1$. These periodic oscillations correspond to the first type of spiking generated by chargedischarge cycles in the circuit. As $V_{e x t}$ further increases this spiking regime ceases out as the result of the saddle-node bifurcation at $V_{e x t} \approx 239.9$. Thus, the mechanisms for the onset of the first type of spiking is associated with Andronov-Hopf and saddle-node bifurcations for the limit cycles. These mechanisms are consistent with the generation of charge-discharge spiking in other types of memristive circuits, e.g. based on $\mathrm{Ni}_{2} \mathrm{O}_{x}$ memristors previously analyzed in Ref. ${ }^{22}$.

The middle branch demonstrates an Andronov-Hopf bifurcation at $V_{e x t} \approx 203.6$, which for decreasing/increasing $V_{e x t}$ corresponds to the appearance /disappearance of stable periodic solutions. With a decrease of $V_{e x t}$ this limit cycle disappears through the saddle-node bifurcation at $V_{e x t} \approx 186.7$. For $V_{\text {ext }}>203.6$ the fixed point of this branch remains stable until $V_{\text {ext }} \approx 280.4$, where it becomes unstable through another Andronov-Hopf bifurcation, which originates an unstable periodic solution (pink dots). This unstable orbit eventually touches the unstable fixed point, manifesting a homoclinic bifurcation $^{51-53}$ and as such the orbit disappears. This branch of fixed points stops its existence at $V_{\text {ext }} \approx 282.1$, where it collides with a branch of unstable fixed points implying a saddle node-bifurcation. The latter branch of unstable fixed points involve in another saddle-node bifurcation at $V_{\text {ext }} \approx 35.3$, which induces an upper branch of stable fixed point. For $V_{\text {ext }}>35.3$ these equilibria are stable and represent a high-resistance regime to which the system switches when the $V_{\text {ext }}$ is gradually increased.

Note, the unstable limit cycles shown in the diagram by pink color points are induced either through the saddlenode bifurcation or through global instabilities including that shown by the gray circle homoclinic bifurcation, which manifest themselves in an abrupt appearance and disappearance of finite-amplitude periodic solutions.

Figure 3 compares the stable self-oscillations (green points in Fig. 2) corresponding to the lower and middle branches for the same value of $V_{\text {ext }}=186.8$. The corresponding projections of phase trajectories are shown in (a). The blue curve, corresponding to the lower branch, demonstrates large oscillations of the voltage across the memristor $V$, which reflects the charge-discharge cycles defined by the capacitance in the circuit (see Fig. 1(a)). This limit cycle corresponds to fast uniform oscillations of $I(t)$ as illustrated in (b). The stable limit cycle born in the middle branch is shown in (a) by the red curve, while the corresponding current oscillations are illustrated in (c). In contrast to the previous case, these oscillations do not involve the large variation of $V$, and their periodicity is rather defined by a "cooling-heating" cycle. Since the temperature changes are quite inertial, these oscillations are slower than charge-discharge oscillations [compare (b) and (c)]. Thus, the middle branch produces the stable selfoscillations representing the second type of spiking.

Remarkably, that previously it has been shown that "cooling-heating" type of spiking is essentially a noiseinduced phenomenon ${ }^{17}$. However, our approach allowed us to grasp it using a purely deterministic model. This suggests an interpretation of the mechanisms for the onset of such spiking in terms of bifurcation theory. In particular, our results suggests that the mechanisms for the appearance of the second type of spiking in diffusive memristors can be interpreted as a saddle- node and Andronov-Hopf bifurcations for noiseinduced cycles.

Our further study showed that the revealed bifurcation mechanisms of the first and the second type of spiking are robust against the variation of the memristor parameters. However, in certain ranges new complex dynamical regimes can appear in the system, including ones that resembles chaotic oscillations. An example of such dynamics is shown in Fig. 4 which was calculated for $M=0.003$ and $V_{e x t}=186.8$. The current oscillations in such a regime illustrated in (a), demonstrate spiking behavior with quite random variations of the spike heights and rather erratic fast background oscillations near $I \approx 0.2$. The chaotic character of such dynamics becomes even more evident in the projection of the corresponding phase trajectory depicted in (b). Here, the current spikes correspond to long irregular excursions in the phase space, while the background oscillations are represented by fast chaotic rotations in the vicinity of $x_{0} \approx-0.65$. The large fluctuations of $V$ suggests that this regime involves a charge-discharge mechanism.

The detailed analysis of such dynamics is beyond the scope of this paper and will be a subject of further investigation. Remarkably though, that some types of the memristive devices have been known to show chaotic phenomena see, e.g., Ref. ${ }^{54}$. However, the diffusive memristors are essentially stochastic 

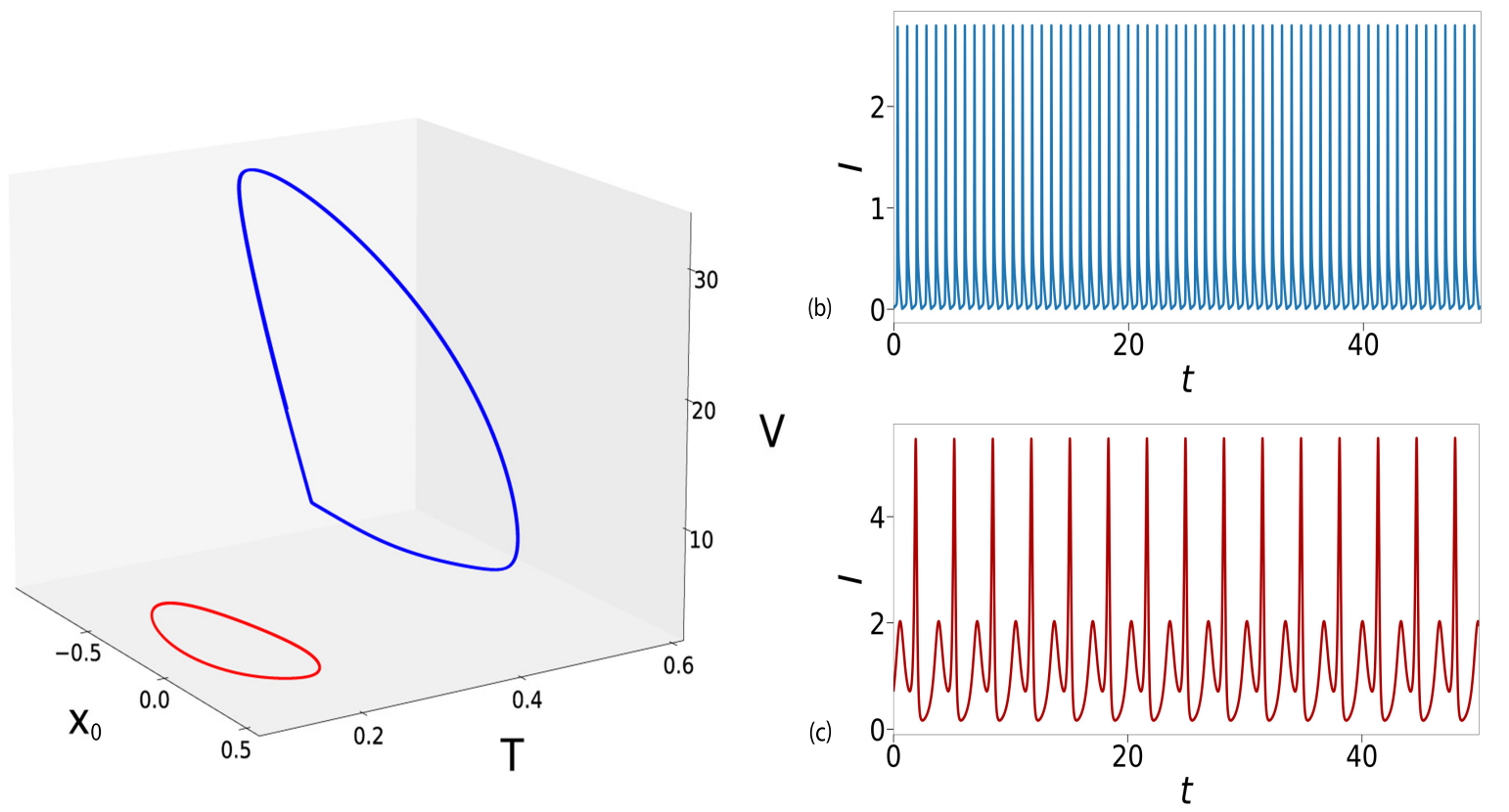

FIG. 3: (a) Phase space of $x_{0}, T$ and $V$ corresponding to the coexistence of two limit cycles at $V_{\text {ext }}=186.8$. The blue line corresponding to the first form of spiking, the red line corresponding to the second spiking mode. (b) Time realization $I(t)$ corresponding to the self sustained periodic oscillations due to charge-discharge effects. (c) Time realization $I(t)$ corresponding to the self-sustained periodic oscillations due to heating-cooling effects.

devices, in which the deterministic chaos, if it exists, is practically impossible to register because of the presence of a nonnegligible noise. Our results suggest that under certain conditions this device can demonstrate chaos-like dynamics in the statistical description, similarly to the phenomena previously reported for ensemble of noisy oscillators ${ }^{55}$.

\section{CONCLUSION}

In this paper we present an approach to model stochastic phenomena in circuits involving diffusive memristors in a deterministic model. The electric properties of these devices are determined by $\mathrm{Ag}$ clusters drifting between the terminals under the action of an electric field, heat exchange with the de-

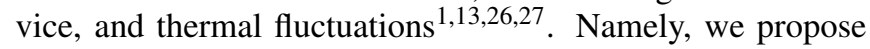
a model of the diffusive memristor where the Langevin equation describing the motion of an $\mathrm{Ag}$-metallic nanocluster is replaced by its Fokker-Planck counterpart. Approximating a solution of the Fokker-Planck equation by a Gaussian distribution allows us to obtain a set of deterministic equations for the average particle position and the width of the distribution related to cluster fluctuations. Our analysis of the obtained set of ordinary differential equations shows that the model allows for describing two different spiking regimes which were originally obtained in the full stochastic simulation ${ }^{17}$ of the original set of stochastic equations (1). We investigated this new system and showed that the predominant mechanisms that lead to the onset of different spiking regimes is associated with
Andronov-Hopf bifurcations and saddle-node bifurcations for limit cycles. We also demonstrated that for certain parameters the new model can demonstrate chaos-like dynamics. The possibility to describe the mechanisms of stochastic spiking in diffusive memristors in terms of bifurcation and chaos theories paves a new avenue for informing the design of memristive circuits and controlling their dynamical regimes. The latter is crucially important for the development of memristive systems for artificial intelligence and neuromorphic computers.

\section{ACKNOWLEDGMENTS}

This work was supported by The Engineering and Physical Sciences Research Council (EPSRC) (grant No $\mathrm{EP} / \mathrm{S} 032843 / 1)$.

\section{DATA AVAILABILITY}

The data that support the findings of this study are available from the corresponding author upon reasonable request.

\footnotetext{
${ }^{1}$ Z. Wang, S. Joshi, S. E. Savel'ev, H. Jiang, R. Midya, P. Lin, M. Hu, N. Ge, J. P. Strachan, Z. Li, et al., "Memristors with diffusive dynamics as synaptic emulators for neuromorphic computing," Nature Materials 16, 101 (2017). ${ }^{2}$ M.-W. Hsu, S. Lessmann, M.-C. Sung, T. Ma, and J. E. Johnson, "Bridging the divide in financial market forecasting: machine learners vs. financial economists," Expert Systems with Applications 61, 215-234 (2016).
} 


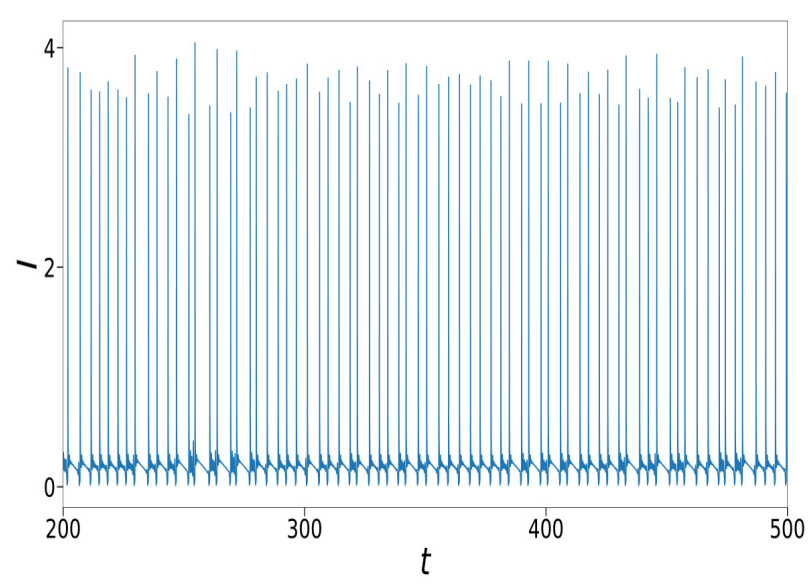

(a)

FIG. 4: Chaotic time realization of $I(t)$ (a) and the corresponding projection of the phase trajectory (b) at $M=0.003$.
${ }^{3}$ A. Radovic, M. Williams, D. Rousseau, M. Kagan, D. Bonacorsi, A. Himmel, A. Aurisano, K. Terao, and T. Wongjirad, "Machine learning at the energy and intensity frontiers of particle physics," Nature 560, 41 (2018).

${ }^{4}$ G. Carleo, I. Cirac, K. Cranmer, L. Daudet, M. Schuld, N. Tishby, L. VogtMaranto, and L. Zdeborová, "Machine learning and the physical sciences," Rev. Mod. Phys. 91, 045002 (2019).

${ }^{5}$ Y. Ma, Z. Wang, H. Yang, and L. Yang, "Artificial intelligence applications in the development of autonomous vehicles: a survey," IEEE/CAA Journal of Automatica Sinica 7, 315-329 (2020).

${ }^{6}$ I. Yaqoob, L. U. Khan, S. M. A. Kazmi, M. Imran, N. Guizani, and C. S. Hong, "Autonomous driving cars in smart cities: Recent advances, requirements, and challenges," IEEE Network 34, 174-181 (2020).

${ }^{7}$ R. Schaller, "Moore's law: past, present and future," IEEE Spectrum 34, 52-59 (1997).

${ }^{8}$ L. Chua, "Memristor-the missing circuit element," IEEE Trans. Circuit Theory CT-18, 507 (1971).

${ }^{9}$ L. Chua and S. Kang, "Memristive devices and systems," Proc. IEEE 64, 209 (1976).

${ }^{10}$ D. B. Strukov, G. S. Snider, D. R. Stewart, and R. S. Williams, "The missing memristor found," Nature 453, 80-83 (2008).

${ }^{11} \mathrm{G}$. Huang and Y. Ho, in Advances in Non-volatile Memory and Storage Technology, edited by Y. Nishi (Woodhead Publishing, 2014) pp. 370-397.

${ }^{12}$ R. Wang, J.-Q. Yang, J.-Y. Mao, Z.-P. Wang, S. Wu, M. Zhou, T. Chen, Y. Zhou, and S.-T. Han, "Recent advances of volatile memristors: Devices, mechanisms, and applications," Advanced Intelligent Systems 2, 2000055 (2020).

${ }^{13}$ Z. Wang, S. Joshi, S. Savel'ev, W. Song, R. Midya, Y. Li, M. Rao, P. Yan, S. Asapu, Y. Zhuo, H. Jiang, P. Lin, C. Li, J. H. Yoon, N. K. Upadhyay, J. Zhang, M. Hu, J. P. Strachan, M. Barnell, Q. Wu, H. Wu, R. S. Williams, Q. Xia, and J. J. Yang, "Fully memristive neural networks for pattern classification with unsupervised learning," Nature Electronics 1, 137-145 (2018).

${ }^{14}$ Q. Hua, H. Wu, B. Gao, M. Zhao, Y. Li, X. Li, X. Hou, M.-F. (Marvin) Chang, P. Zhou, and H. Qian, "A threshold switching selector based on highly ordered ag nanodots for x-point memory applications," Advanced Science 6, 1900024 (2019).

${ }^{15} \mathrm{~S}$. Saitoh and K. Kinoshita, "Oxide-based selector with trap-fillingcontrolled threshold switching," Applied Physics Letters 116, 112101 (2020).
${ }^{16}$ W. Yi, K. K. Tsang, S. K. Lam, X. Bai, J. A. Crowell, and E. A. Flores, "Biological plausibility and stochasticity in scalable vo2 active memristor neurons," Nat Commun 9, 4661 (2018).

${ }^{17}$ Y. Ushakov, A. Balanov, and S. Savel'ev, "Role of noise in spiking dynamics of diffusive memristor driven by heating-cooling cycles," Chaos, Solitons \& Fractals 145, 110803 (2021).

${ }^{18}$ A. Wojtusiak, A. Balanov, and S. Savel'ev, "Intermittent and metastable chaos in a memristive artificial neuron with inertia," Chaos, Solitons \& Fractals 142, 110383 (2021).

${ }^{19}$ X. Zhang, Y. Zhuo, Q. Luo, Z. Wu, R. Midya, Z. Wang, W. Song, R. Wang, N. K. Upadhyay, Y. Fang, F. Kiani, M. Rao, Y. Yang, Q. Xia, Q. Liu, M. Liu, and J. J. Yang, "An artificial spiking afferent nerve based on mott memristors for neurorobotics," Nature Communications 11, 51 (2020).

${ }^{20}$ S. Kumar, R. Williams, and Z. Wang, "Third-order nanocircuit elements for neuromorphic engineering," Nature 585, 518 (2020).

${ }^{21} \mathrm{X}$. Zhang and K. Long, "Improved learning experience memristor model and application as neural network synapse," IEEE Access 7, 15262-15271 (2019).

${ }^{22}$ B. Johnson, K. Brahim, A. Balanov, S. Savel'ev, and P. Borisov, "Transition from noise-induced to self-sustained current spiking generated by a nbox thin film threshold switch," Applied Physics Letters 118, 023502 (2021).

${ }^{23}$ D. Vodenicarevic, N. Locatelli, F. A. Araujo, J. Grollier, and D. Querlioz, "A nanotechnology-ready computing scheme based on a weakly coupled oscillator network," Sci Rep 7, 44772 (2017).

${ }^{24}$ G. Csaba and W. Porod, "Coupled oscillators for computing: A review and perspective," Applied Physics Reviews 7, 011302 (2020).

${ }^{25}$ M. L. Simpson, C. D. Cox, M. S. Allen, J. M. McCollum, R. D. Dar, D. K. Karig, and J. F. Cooke, "Noise in biological circuits," WIREs Nanomedicine and Nanobiotechnology 1, 214-225 (2009).

${ }^{26}$ H. Jiang, D. Belkin, S. E. Savel'ev, S. Lin, Z. Wang, Y. Li, S. Joshi, R. Midya, C. Li, M. Rao, M. Barnell, Q. Wu, J. J. Yang, and Q. Xia, “A novel true random number generator based on a stochastic diffusive memristor," Nature Communications 8, 882 (2017).

${ }^{27}$ R. Midya, Z. Wang, J. Zhang, S. E. Savel'ev, C. Li, M. Rao, M. H. Jang, S. Joshi, H. Jiang, P. Lin, K. Norris, N. Ge, Q. Wu, Z. Barnell, M. amd Li, H. L. Xin, R. S. Williams, Q. Xia, and J. J. Yang, "Anatomy of ag/hafniabased selectors with 1010 nonlinearity," Adv. Mater. 29, 1604457 (2017).

${ }^{28}$ S. E. Savel'ev, A. S. Alexandrov, A. M. Bratkovsky, and R. S. Williams, "Molecular dynamics simulations of oxide memory resistors (memristors)," 
Nanotechnology 22, 254011 (2011).

${ }^{29}$ S. E. Savel'ev, A. S. Alexandrov, A. M. Bratkovsky, and R. S. Williams, "Molecular dynamics simulations of oxide memristors: thermal effects," Applied Physics A 102, 891 (2011).

${ }^{30}$ W. Yi, S. Savel'ev, G. Medeiros-Ribeiro, F. Miao, M.-X. Zhang, J. Yang, A. Bratkovsky, and R. Williams, "Molecular dynamics simulations of oxide memristors: Crystal field effects," Nature Communications volume 7, 11142 (2016)

${ }^{31}$ S. E. Savel'ev, A. S. Alexandrov, A. M. Bratkovsky, and R. S. Williams, "Molecular dynamics simulations of oxide memristors: Crystal field effects," Applied Physics Letters 99, 053108 (2011).

${ }^{32}$ S. Kumar, R. S. Williams, and Z. Wang, "Third-order nanocircuit elements for neuromorphic engineering," Nature 585, 518-523 (2020).

${ }^{33}$ S. Kumar, J. P. Strachan, and R. S. Williams, "Chaotic dynamics in nanoscale nbo $_{2}$ mott memristors for analogue computing," Nature 548, 318-321 (2017).

${ }^{34}$ I. Messaris, R. Tetzlaff, A. Ascoli, R. Williams, S. Kumar, and L. Chua, "A simplified model for a nbo2 mott memristor physical realization," in 2020 IEEE International Symposium on Circuits and Systems (ISCAS) (IEEE, 2020) pp. 1-5.

${ }^{35}$ V. R. Misko, S. Savel'ev, A. L. Rakhmanov, and F. Nori, "Negative differential resistivity in superconductors with periodic arrays of pinning sites," Physical Review B 75, 024509 (2007).

${ }^{36}$ Y. Ushakov, A. Akther, P. Borisov, D. Pattnaik, S. Savel'ev, and A. Balanov, "Deterministic mechanisms of spiking in diffusive memristors," Chaos, Solitons \& Fractals 149, 110997 (2021).

${ }^{37}$ H. Goldstein, C. Poole, and J. Safko, Classical Mechanics, 3rd ed. (Pearson, 2001).

${ }^{38}$ A. S. Alexandrov, A. M. Bratkovsky, B. Bridle, S. E. Savel'ev, D. B. Strukov, and R. S. Williams, "Current-controlled negative differential resistance due to joule heating in tio2," Appl. Phys. Lett. 99, 202104 (2011).

${ }^{39}$ A. V. Moskalenko, S. N. Gordeev, O. F. Koentjoro, P. R. Raithby, R. W. French, F. Marken, and S. E. Savel'ev, "Nanomechanical electron shuttle consisting of a gold nanoparticle embedded within the gap between two gold electrodes," Physical Review B 79, 241403 (2009).

${ }^{40}$ S. E. Savel'ev, F. Marchesoni, and A. M. Bratkovsky, "Mesoscopic resistive switch: non-volatility, hysteresis and negative differential resistance," The European Physical Journal B volume 86, 501 (2013).

${ }^{41}$ V. S. Anishchenko, A. B. Neiman, and M. A. Safanova, "Stochastic resonance in chaotic systems," Journal of Statistical Physics 70, 183-196 (1993).

${ }^{42}$ V. S. Anishchenko, A. B. Neiman, A. N. Silchenko, and I. A. Khovanov, "Phase synchronization of switchings in stochastic and chaotic bistable sys- tems," Dynamics and Stability of Systems 14, 211-231 (1999).

${ }^{43}$ V. Anishchenko, V. Astakhov, A. Neiman, T. Vadivasova, and L. Schimansky-Geier, Nonlinear Dynamics of Chaotic and Stochastic Systems: Tutorial and Modern Developments, Springer Series in Synergetics (Springer Berlin Heidelberg, 2007).

${ }^{44}$ S. Astakhov, A. Feoktistov, V. S. Anishchenko, and J. Kurths, "Synchronization of multi-frequency noise-induced oscillations," Chaos: An Interdisciplinary Journal of Nonlinear Science 21, 047513 (2011).

${ }^{45}$ N. Semenova, A. Zakharova, V. Anishchenko, and E. Schöll, "Coherenceresonance chimeras in a network of excitable elements," Phys. Rev. Lett. 117, 014102 (2016)

${ }^{46}$ V. S. Anishchenko, T. E. Vadivasova, J. Kurths, G. A. Okrokvertskhov, and G. I. Strelkova, "Autocorrelation function and spectral linewidth of spiral chaos in a physical experiment," Phys. Rev. E 69, 036215 (2004).

${ }^{47}$ V. S. Anishchenko, T. E. Vadivasova, G. A. Okrokvertskhov, and G. I. Strelkova, "Statistical properties of dynamical chaos," Physics-Uspekhi 48, 151-166 (2005)

${ }^{48}$ V. S. Anishchenko and H. Herzel, "Noise-induced chaos in a system with homoclinic points," ZAMM - Journal of Applied Mathematics and Mechanics / Zeitschrift für Angewandte Mathematik und Mechanik 68, 317-318 (1988).

${ }^{49}$ L. Petzold, "Automatic selection of methods for solving stiff and nonstiff systems of ordinary differential equations," SIAM Journal on Scientific and Statistical Computing 4, 136-148 (1983).

${ }^{50} \mathrm{~S}$. Pearson and H. Anson, "Demonstration of some electrical properties of neon-filled lamps," Proceedings of the Physical Society of London 34, 175 (1921).

${ }^{51}$ J. Guckenheimer and P. J. Holmes, Nonlinear Oscillations, Dynamical Systems, and Bifurcations of Vector Fields (Springer-Verlag New York, 1983).

${ }^{52}$ V. S. Anishchenko, Dynamical Chaos - Models and Experiments. Appearance Routes and Structure of Chaos in Simple Dynamical Systems (World Scientific Publishing, Singapore, 1995).

${ }^{53}$ Y. Kuznetsov, Elements of Applied Bifurcation Theory (Springer-Verlag New York, 2004).

${ }^{54}$ S. Kumar, J. Strachan, and R. Williams, "Chaotic dynamics in nanoscale nbo2 mott memristors for analogue computing," Nature 548, 318-321 (2017).

${ }^{55}$ M. A. Zaks, A. B. Neiman, S. Feistel, and L. Schimansky-Geier, "Noisecontrolled oscillations and their bifurcations in coupled phase oscillators," Phys. Rev. E 68, 066206 (2003). 